\title{
Screening hub genes in coronary artery disease based on integrated analysis
}

\author{
Fei Long ${ }^{1}$, Ling Wang ${ }^{1}$, Lei Yang ${ }^{1}$, Zhou Ji ${ }^{1}$, YaGuang $\mathrm{Hu}^{1}$ \\ ${ }^{1}$ Department of Cardiology, The Third Affiliated Hospital of Jinzhou Medical University, \\ Liaoning Province, China
}

\begin{abstract}
Background: Coronary artery disease (CAD) is the leading cause of mortality worldwide. Identifying key pathogenic genes benefits the understanding molecular mechanism of CAD.

Methods: In this study, 5 microarray data sets from the blood sample of 312 CADs and 277 healthy controls were downloaded. Limma and metaMA packages were used to identify differentially expressed genes. The functional enrichment analysis of differentially expressed genes was further performed by Gene Ontology and Kyoto Encyclopedia of Genes and Genomes. Additionally, protein-protein interaction and transcript factors-target networks were performed based on top $10 \mathrm{up}$ - and down-regulated differentially expressed genes to further study the biological function. Last, real-time quantitative polymerase chain reaction ( $R T-q P C R$ ) was used to validate the integrated analysis result.

Results: A total of 528 differentially expressed genes were obtained. All differentially expressed genes were significantly involved in signal transduction and the MAPK signaling pathway. Among MAPK signaling pathway, IL1R2, ARRB2 and PRKX were associated with CAD. Furthermore, there were 4 common differentially expressed genes including PLAUR, HSPH1, ZMYND11 and S100A8 in the protein-protein interaction and transcript factors-target networks, which played crucial roles in the development of CAD. In quantitative RT-qPCR, the expression of PRKX, HSPH1 and ZMYND11 was down-regulated and consistent with the integrated analysis.

Conclusions: Identified 7 differentially expressed genes (IL1R2, ARRB2, PRKX, PLAUR, HSPH1, ZMYND11 and S100A8) may play crucial roles in the development of CAD. (Cardiol J 2018; 25, 3: 403-411)

Key words: coronary artery disease, differentially expressed gene, protein-protein interaction, transcript factors
\end{abstract}

\section{Introduction}

Coronary artery disease (CAD) is a heart disease with atherosclerosis lesion and leads to vessel blockage, myocardial ischemia, hypoxia and necrosis. It is a major health problem all over the world and is supposed to be the primary cause of death by $2020[1,2]$. Atherosclerosis is the main cause of CAD and the process begins early in a progressive interaction between genetic factors and the environment [3]. The metabolic syndrome, also present in $\mathrm{CAD}$, is defined as a number of risk factors including obesity, hypertension, dyslipidemia and hyperglycemia [3]. Additionally, some risk factors such as age, sex and smoking are linked to CAD [4].

Up to now, with advances in both percutaneous coronary intervention and coronary artery bypass graft surgery, mortality from CAD has remarkably decreased [5]. However, the poor prognosis for patients with $\mathrm{CAD}$ and unclear pathology mechanism of CAD is still a hot topic. Therefore, identifying key

Address for correspondence: Dr. YaGuang Hu, Department of Cardiology, The Third Affiliated Hospital of Jinzhou Medical University, No. 2, Section.5, Linghe District, Jinzhou, Liaoning Province 121000, China, tel: (+86)-0416-3999016, fax: (+86)-0416-3999016, e-mail: lnslzg@163.com 
Table 1. Five microarray data sets.

\begin{tabular}{lllcc}
\hline GEO accession & Author & Platform & Samples (P:N) & Year \\
\hline GSE71226 & Meng F & GPL570[HG-U133_Plus_2] Affymetrix Human & $3: 3$ & 2015 \\
GSE42148 & Arvind P & $\begin{array}{l}\text { GPL13607 Agilent-028004 SurePrint G3 Human GE } \\
\text { 8x60K Microarray (Feature Number version) }\end{array}$ & $13: 11$ & 2012 \\
GSE20681 & Biros E & $\begin{array}{l}\text { GPL4133Agilent-014850 Whole Human Genome } \\
\text { Microarray 4x44K G4112F (Feature Number version) }\end{array}$ & $99: 99$ & 2011 \\
GSE20680 & Wingrove JA & $\begin{array}{l}\text { GPL4133Agilent-014850 Whole Human Genome } \\
\text { Microarray 4x44K G4112F (Feature Number version) }\end{array}$ & $87: 52$ & 2011 \\
GSE12288 & Grass P & $\begin{array}{l}\text { GP96 [HG-U133A] Affymetrix Human Genome } \\
\text { U133A Array }\end{array}$ & $110: 112$ & 2009 \\
\hline
\end{tabular}

genes in patients with $\mathrm{CAD}$ could facilitate a better understanding of the important role in the process of $\mathrm{CAD}$, which will further help provide the potential molecular mechanism in the development of CAD.

Using the microarray data analysis, Yang et al. [6] identified the biomarkers for ischemic cardiomyopathy. It is noted that high throughput sequencing is a fast and comprehensive method of studying various diseases. In this study, the transcription data of CAD was integrated and analyzed identifying 528 differentially expressed genes (DEGs) including 310 up-regulated and 218 downregulated genes. Gene Ontology (GO) and Kyoto Encyclopedia of Genes and Genomes (KEGG) were further used to annotate function of identified DEGs. In order to further study the regulatory roles of DEGs, protein-protein interaction (PPI) and transcript factors (TFs)-target networks were performed. At last, 7 key DEGs (IL1R2, ARRB2, PRKX, PLAUR, HSPH1, ZMYND11 and S100A8) and MAPK signaling pathways were identified, which may play vital roles in the process of CAD.

\section{Methods}

\section{Microarray data}

In this study, the keywords were used: ("coronary disease" [MeSH Terms] OR coronary disease [All Fields]) AND "Homo sapiens" [porn] AND "gse" [Filter] AND "Expression profiling by array" [Filter]. Then, five microarray data sets including GSE71226, GSE42148, GSE20681, GSE20680 and GSE12288 were downloaded from the GEO database (http://www.ncbi.nlm.nih.gov/geo/). And, all selected datasets were transcription data of the blood samples (no drug stimulation or transfected treatment) from 312 CAD and 277 healthy controls. The control groups of these databases were properly matched. Additionally, age and sex between case and normal groups were properly matched. There were no comorbidities in both case and normal groups. Detailed information of five microarray data sets is listed in Table 1.

\section{Differentially expressed genes identification and functional enrichment analysis}

The data was first preprocessed by removing the probes that corresponded to multiple genes. Among genes with multiple corresponding probes, only those genes with the highest average expression were reserved. Then, 20468 genes in GSE71226, 19726 genes in GSE42148, 19751 genes in GSE20681, 19751 genes in GSE20680 and 19751 genes in GSE12288 were obtained and 12013 genes were identified by intersected above reserved genes. Limma and metaMA packages were further applied to identify DEGs and an inverse normal method was used to combine the $p$ value [7]. Finally, candidate DEGs were chosen with a cut-off $p$ value of $<0.05$. In order to study the biological characteristics of the DEGs, enrichment analysis was conducted by GeneCoDis. All DEGs were annotated and built on the GO (http:// geneontology.org/) and the KEGG [8] dataset.

\section{Protein-protein interaction network analysis}

It is a known fact that proteins and other molecules were not playing roles alonebut interact with each other and form a network, which determines the characteristic of cells, tissue and individuals. It may result in dysfunction of the cell upon this network and is thus destroyed. In order to explore the molecular mechanism of CAD, PPI was performed based on the dataset of BioGRID [9] and the network was constructed using Cytoscape [10]. Selected genes that had a large number of interactions with other genes were considered to have more important roles in the pathogenesis. And the degree of a gene 
was the number of the exhibited interactions. Gene with a degree of $>10$ was considered as a critical hub molecule.

\section{Transcript factors screening of DEGs}

Transcript factors can regulate the expression of various genes by binding to the specific DNA sequence in the promoter of target genes. In order to further study the molecular mechanism of CAD, the upstream promoter $2 \mathrm{~KB}$ sequence of top $10 \mathrm{up}$ and down-regulated DEGs was first downloaded from the UCSC website. Then, the tool of match in the TRANSFAC website was used to identify the TFs. Last, the regulation network between TFs and DEGs was established through Cytoscape software. Selected genes that had a large number of interactions with TFs were considered to play more significant roles in the pathogenesis. And the gene degree was the number of the exhibited interactions. A gene with a degree of $>5$ was considered as a critical hub molecule.

\section{RT-qPCR}

Five patients diagnosed as CAD and 5 health controls were enrolled in this study. Both the $\mathrm{CAD}$ and corresponding normal blood samples were obtained and immediately frozen in liquid nitrogen. Written informed consent was given by CAD patients before enrollment and this study was approved by the ethics committee of the local hospital.

According to manufacturer protocols, total RNA of the blood samples was extracted using the RNAliquid Reagent (Beijing Huitian east technology co., LTD). And the A260/280 ratio was 1.8-2.0, which indicated the purity of RNA extracted. $1 \mu \mathrm{g}$ RNA was applied to synthesize DNA by FastQuant cDNA Reverse Transcriptase (TIANGEN). Then real-time polymerase chain reaction (PCR) was performed in an ABI 7500 real-time PCR system with SuperReal PreMix Plus (SYBR Green) SuperReal Mix (TIANGEN). All reactions were performed in triplicate and GAPDH was considered as a negative control. Comparative gene expressions were analyzed by the log2 (fold change) method.

\section{Results}

\section{Differentially expressed genes selection and functional analysis}

In this study, a total of 528 DEGs were identified. Among which, 310 and 218 genes were upregulated and down-regulated, respectively. The top ten up-regulated genes were LIN7A, MMP9,
MYBPH, MBOAT2, ITPK1, CEACAM4, PLAUR, USB1, CBS and S100A8. And NBEA, ZMYND11, BYSL, DCXR, PRKX, HLA-DPB1, HSPH1, SCRIB, LAGE3 and CROCCP2 were the top 10 down-regulated genes. After cluster analysis, the heat map of top 100 DEGs was presented in Supplementary Figure 1 and the top 100 genes were listed in Supplementary Table 1 . The GO analysis showed that these DEGs were significantly enriched in signal transduction, cell adhesion, positive regulation of apoptosis process of biological process term and protein binding, hydrolase activity, nucleotide binding of molecular function term and cytoplasm, plasma membrane, nucleus of cellular component term. Additionally, these DEGs were remarkably involved in leishmaniasis, leukocyte transendothelial migration, phagosome, amoebiasis and MAPK signal pathway. The GO and KEGG annotation function were shown in Tables 2 and 3, respectively. It is pointed out that there were 13 DEGs (MAP4K2, IL1R2, RASGRP1, DDIT3, FAS, CACNA1H, ARRB2, HSPA6, PAK1, PRKX, MKNK1, MAPK14 and HSPB1) participating in the MAPK signaling pathway. The MAPK signaling pathway is shown in Figure 1.

\section{Protein-protein interaction network analysis}

Protein-protein interaction is crucial to all biological processes [11]. In order to study the biological mechanism of CAD, the PPI involving top 10 (up- and down-regulated) DEGs were established (Suppl. Fig. 2). This network was comprised of node and edge. The node and edge represented the protein and the interaction, respectively. In the network, there were 653 nodes and 688 edges. There were 10 up-regulated and 9 down-regulated DEGs in the network. Among which, the top 8 DEGs with the highest degree were BYSL (degree $=118$ ), PLAUR (degree = 98), HSPH1 (degree = 84), $\mathrm{CBS}($ degree $=64)$, ZMYND11 (degree $=51)$, LAGE3 $($ degree $=45)$, S100A8 $($ degree $=43)$ and SCRIB (degree $=43)$.

\section{Transcript factors regulatory network}

Transcript factors play roles in regulating numbers of genes, such as cytokines, growth factors, cell adhesion molecules and so on. The study of TFs-target genes network is valuable in understanding the regulatory mechanism of biological function. In order to further study the biological roles of top 10 DEGs (up- and downregulated) under the regulation of TFs, TFs-target genes regulatory network was performed (Fig. 2). The network was comprised of 60 nodes and 115 
Table 2. The enriched Gene Ontology (GO) annotation of differentially expressed genes in coronary artery disease.

\begin{tabular}{|c|c|c|c|}
\hline GO ID & GO term & Support & FDR \\
\hline \multicolumn{4}{|c|}{ Biological process } \\
\hline GO:0007165 & Signal transduction & 54 & 3.37E-10 \\
\hline GO:0007155 & Cell adhesion & 33 & 1.47E-08 \\
\hline GO:0043065 & Positive regulation of apoptotic process & 14 & 0.000183 \\
\hline GO:0006954 & Inflammatory response & 17 & 0.000197 \\
\hline GO:0042493 & Response to drug & 18 & 0.000211 \\
\hline GO:0014070 & Response to organic cyclic compound & 11 & 0.000232 \\
\hline GO:0007596 & Blood coagulation & 22 & 0.000352 \\
\hline GO:0006508 & Proteolysis & 24 & 0.000462 \\
\hline GO:0019047 & Provirus integration & 4 & 0.000544 \\
\hline GO:0006955 & Immune response & 19 & 0.000792 \\
\hline \multicolumn{4}{|c|}{ Molecular function } \\
\hline GO:0005515 & Protein binding & 166 & $2.50 \mathrm{E}-28$ \\
\hline GO:0016787 & Hydrolase activity & 50 & 7.03E-12 \\
\hline GO:0000166 & Nucleotide binding & 78 & $1.71 \mathrm{E}-11$ \\
\hline GO:0046872 & Metal ion binding & 87 & $1.60 \mathrm{E}-08$ \\
\hline GO:0005524 & ATP binding & 54 & 1.64E-07 \\
\hline GO:0003824 & Catalytic activity & 23 & $9.48 \mathrm{E}-07$ \\
\hline GO:0004872 & Receptor activity & 49 & $5.96 \mathrm{E}-05$ \\
\hline GO:0005488 & Binding & 28 & 0.000389 \\
\hline GO:0042803 & Protein homodimerization activity & 22 & 0.000651 \\
\hline GO:0005529 & Sugar binding & 12 & 0.000732 \\
\hline GO:0003700 & Sequence-specific DNA binding transcription factor activity & 31 & 0.000779 \\
\hline \multicolumn{4}{|c|}{ Cellular component } \\
\hline GO:0005737 & Cytoplasm & 177 & 3.33E-25 \\
\hline GO:0005886 & Plasma membrane & 126 & 1.19E-18 \\
\hline GO:0005634 & Nucleus & 157 & $8.86 \mathrm{E}-16$ \\
\hline GO:0016020 & Membrane & 128 & 2.96E-15 \\
\hline GO:0005624 & Membrane fraction & 37 & $3.23 \mathrm{E}-13$ \\
\hline GO:0009986 & Cell surface & 27 & 3.84E-12 \\
\hline GO:0005887 & Integral to plasma membrane & 49 & 2.00E-11 \\
\hline GO:0005829 & Cytosol & 77 & $2.11 \mathrm{E}-11$ \\
\hline GO:0016021 & Integral to membrane & 121 & 1.69E-10 \\
\hline GO:0005739 & Mitochondrion & 57 & 1.70E-10 \\
\hline
\end{tabular}

FDR - false discovery rate

edges. A total of 378 interaction pairs were obtained involving 40 TFs. The top 6 TFs that covered the most downstream genes were Oct-1, Evi-1, Pax-4, AP-1, FOXD3 and HNF-3beta. Among which, Oct-1 $($ degree $=11)$, Pax-4 (degree $=8)$ and Evi-1 (degree $=8$ ) were the TFs with the highest degree. At the same time, the top 8 target DEGs with the highest degree were HSPH1 (degree $=12$ ), ZMYND11 (degree $=10)$, S100A8 $($ degree $=9)$, PRKX (degree $=9$ ), PLAUR (degree $=9$ ), LIN7A (degree $=8)$, CEACAM4 (degree $=8)$, DCXR (degree $=7$ ).

\section{RT-qPCR}

Previous study validated the expression of some microRNAs by quantitative polymerase chain reaction in plasma for stable LAD [12]. In this study, 5 patients of CAD and 5 health controls were enrolled in this study for validation. The collected blood samples were used to validate the expression 
Table 3. The enriched Kyoto Encyclopedia of Genes and Genomes (KEGG) annotation of differentially expressed genes in coronary artery disease.

\begin{tabular}{|c|c|c|c|c|}
\hline KEGG ID & KEGG term & Support & FDR & Gene list \\
\hline hsa05140 & Leishmaniasis & 9 & 9.69E-05 & $\begin{array}{l}\text { TLR2, NCF1, ITGAM, TLR4, HLA-DPB1, FCGR2A, } \\
\text { MAPK14, NCF4, NCF2 }\end{array}$ \\
\hline hsa04670 & $\begin{array}{l}\text { Leukocyte transen- } \\
\text { dothelial migration }\end{array}$ & 10 & 0.000426 & $\begin{array}{l}\text { MMP9, CTNNA1, NCF1, CD99, ITGAM, RHOH, VASP, } \\
\text { MAPK14, NCF4, NCF2 }\end{array}$ \\
\hline hsa04145 & Phagosome & 11 & 0.000577 & $\begin{array}{l}\text { CTSS, TLR2, NCF1, ITGAM, TLR4, HLA-DPB1, } \\
\text { FCGR2A, NCF4, NCF2, ATP6V1B2, PLA2R1 }\end{array}$ \\
\hline hsa05146 & Amoebiasis & 9 & 0.000625 & $\begin{array}{l}\text { TLR2, IL1R2, ITGAM, CXCL1, TLR4, ARG1, PRKX, } \\
\text { HSPB1, SERPINB4 }\end{array}$ \\
\hline hsa04380 & $\begin{array}{l}\text { Osteoclast } \\
\text { differentiation }\end{array}$ & 10 & 0.000669 & $\begin{array}{l}\text { NCF1, SIRPA, SIRPB1, LILRB3, FCGR2A, LILRA2, SPI1, } \\
\text { MAPK14, NCF4, NCF2 }\end{array}$ \\
\hline hsa00910 & $\begin{array}{l}\text { Nitrogen } \\
\text { metabolism }\end{array}$ & 5 & 0.000826 & HAL, CA5A, GLUL, CA5B, CA4 \\
\hline hsa04060 & $\begin{array}{l}\text { Cytokine-cytokine } \\
\text { receptor interaction }\end{array}$ & 14 & 0.000908 & $\begin{array}{l}\text { INHBB, TNFRSF10C, IL11, IL1R2, FAS, CXCL1, EDA, } \\
\text { FLT3, HGF, TNFRSF9, FLT3LG, ACVR2A, CD27, CCR3 }\end{array}$ \\
\hline hsa05152 & Tuberculosis & 11 & 0.001210 & $\begin{array}{l}\text { CTSS, TLR2, ITGAM, TLR4, HLA-DPB1, FCGR2A, } \\
\text { MAPK14, HSPD1, TLR1, LSP1, PLA2R1 }\end{array}$ \\
\hline hsa04110 & Cell cycle & 9 & 0.0018249 & $\begin{array}{l}\text { MDM2, CDC7, MCM7, CCND2, CDK4, CDKN2B, } \\
\text { TFDP1, MCM3, CDKN2D }\end{array}$ \\
\hline hsa05162 & Measles & 9 & 0.002509 & $\begin{array}{l}\text { TNFRSF10C, TLR2, CCND2, CDK4, STAT3, FAS, TLR4, } \\
\text { HSPA6, PRKCQ }\end{array}$ \\
\hline hsa04010 & $\begin{array}{l}\text { MAPK signaling } \\
\text { pathway }\end{array}$ & 13 & 0.002564 & $\begin{array}{l}\text { MAP4K2, IL1R2, RASGRP1, DDIT3, FAS, CACNA1H, } \\
\text { ARRB2, HSPA6, PAK1, PRKX, MKNK1, MAPK14, } \\
\text { HSPB1 }\end{array}$ \\
\hline
\end{tabular}

FDR - false discovery rate

of integrated analysis. PRKX, HSPH1, ZMYND11 and MMP9 were randomly selected. The real-time quantitative polymerase chain reaction (RT-qPCR) results showed that PRKX, HSPH1 and ZMYND11 were down-regulated, which obtained consistent data with integrated analysis. While MMP9 was down-regulated, which were not in accordance with the integrated analysis result. The consequence is shown in Figure 3.

\section{Discussion}

Coronary artery disease is the leading killer and accounts for one-third of all patient deaths in the world [13]. It is noted that gene expression profiling is an important way to investigate the underlying pathology of $\mathrm{CAD}$. In the present study, integration analysis of transcription data in $\mathrm{CAD}$ was performed. Five microarray data sets were downloaded from the GEO dataset. GO and KEGG annotation analysis was further used to investigate the biological function of these DEGs. Additionally, PPI interaction network and TFstarget genes analysis were utilized to explore the underlying regulatory mechanism of DEGs in
CAD. Finally, RT-qPCR was used to validate the integrated analysis.

In KEGG functional annotation analysis, it was found that there were 13 DEGs, such as IL1R2, ARRB2 and PRKX involved in the MAPK signaling pathway. MAPK is serine/threonine kinase, which is activated in response to various signals. Furthermore, MAPK is associated with a large number of biological processes including cell proliferation, cell apoptosis, cell transformation and vast cell signaling pathways [14]. Interleukin 1 receptor type 2 (IL1R2) is an anti-inflammatory gene [15]. It is pointed out that the expression of IL1R2 is remarkably up-regulated in ST-segment elevation myocardial infarction [16]. It is also upregulated in peripheral blood mononuclear cells and whole blood in patients with advanced heart failure [17]. Additionally, it is involved in the p38 MAPK signaling pathway in patients with cardioembolic and large vessel atherosclerotic stroke [18]. It is worth mentioning that IL1R2 is found to be related to CAD [19]. Thus it can be seen that IL1R2 plays an important role in heart development. In this study, it wasfound that IL1R2 was up-regulated in CAD and participated in the MAPK signaling 


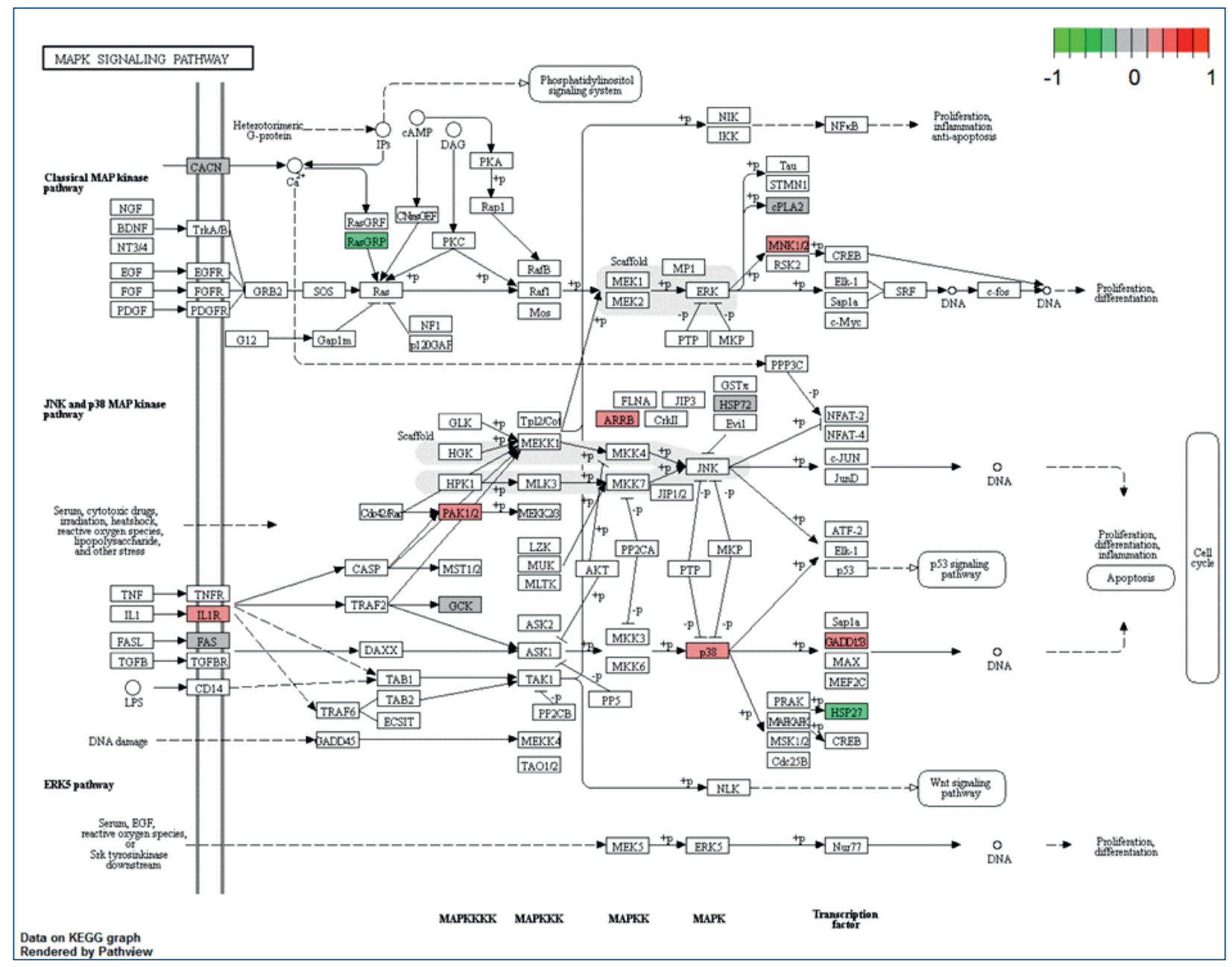

Figure 1. The significantly enriched Kyoto Encyclopedia of Genes and Genomes pathway of differentially expressed genes in coronary artery disease. Color diamonds represent the differentially expressed genes in coronary artery disease.

pathway. This further demonstrated the relationship between IL1R2 and CAD, which indicated that IL1R2 may function by the MAPK signaling pathway in CAD.

The protein encoded by arrestin beta 2 (ARRB2) belongs to the arrestin/beta-arrestin protein family [20]. It is the most obviously altered gene in chronic thromboembolic pulmonary hypertension [21]. It was found that ARRB2 is involved in the inflammation system of the MAPK signaling pathway in patients with atrial fibrillation [22]. Additionally, it was reported that ARRB2 is a hub gene with a degree of $>10$ in the PPI network in $\mathrm{CAD}$ [20]. In the present study, it was found that the expression of ARRB2 was increased in CAD and significantly enriched in the signaling pathway of MAPK. This suggested that ARRB2 may play crucial roles in the process of CAD.
The human protein kinase, X-linked (PRKX) is a member of the family of cAMP-dependent serine threonine kinases. It has been noted that PRKX is enriched in the MAPK signaling pathway in patients with atrial fibrillation [23]. In this study, it was found that the expression of PRKX was down-regulated in CAD and RT-qPCR validated this expression. In addition, PRKX remarkably participated in the signaling pathway of MAPK and was also a downstream gene in a higher degree in the network of TFs-target gene in CAD. This suggested that PRKX may play an important regulatory role in the development of CAD.

In the PPI network and TFs-target regulatory network, we found 4 common hub DEGs including PLAUR, HSPH1, ZMYND11 and S100A8, all of which were top 10 DEGs and played a vital role in CAD. Plasminogen activator, urokinase receptor (PLAUR) 


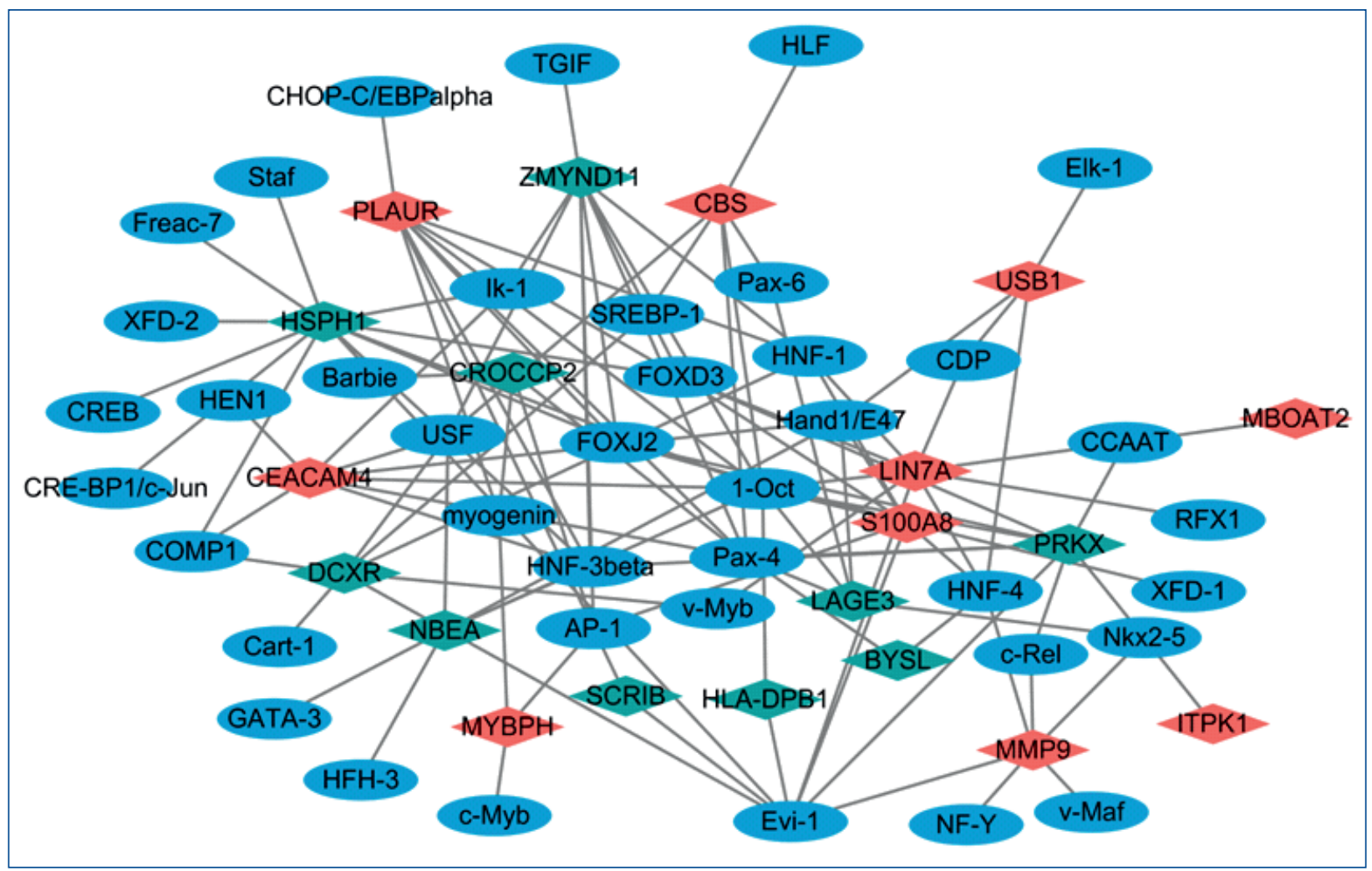

Figure 2. Transcript factors-target genes regulatory network in coronary artery disease. Rhombus and ellipses represent the genes and transcript factors, respectively. The red and green colors represent up-regulation and downregulation, respectively. The gray line indicates the interaction between gene and transcript factors.



Figure 3. Relative expression levels of PRKX, MMP9, $\mathrm{HSPH} 1$, and ZMYND11 at the validation stage in patients with coronary artery disease $(n=5)$ and controls $(n=5)$. The expression level was presented as $2^{-\Delta \Delta c t ;}{ }^{*} p<0.05$; ${ }^{*} p<0.01$.

is a platelet-related angiogenesis gene associated with left ventricular function [24]. It is found differentially expressed in acute myocardial infarction [25].
In addition, the up-regulated expression of PLAUR has been implicated in vascular homeostasis and pathology of peripheral arterial disease [26, 27]. Interestingly, PLAUR is a crucial atherogenic gene and is considered the marker of unstable human atheroma $[28,29]$. Furthermore, it expresses in patients with $\mathrm{CAD}$ and is regarded a therapeutic target for $\mathrm{CAD}[30$, 31]. In this study, increased expression of PLAUR in CAD was found, which further demonstrated the role of PLAUR in the process of CAD.

Heat shock protein family $\mathrm{H}(\mathrm{HSPH})$ member 1 (HSPH1) is a cardiac age-dependent protein [32]. In this study, the expression of HSPH1 was decreased in CAD and RT-qPCR also consistent with the integrated analysis. Additionally, HSPH1 was involved in the PPI and TFs-target network, which suggested the role of HSPH1 in CAD. Zinc finger MYND-type containing 11 (ZMYND11) is a candidate tumor suppressor and expresses in diverse tissues [33, 34]. It is found that ZMYND11 plays critical roles in repressing tumor cell transcriptional process and the low expression level of ZMYND11 in patients with breast cancer is associated with worse prognosis $[35,36]$. In the present 
study, we found the down-regulated expression of ZMYND11 and RT-qPCR also validated the expression. This indicated that ZMYND11 may be involved in CAD.

S100 calcium binding protein A8 (S100A8) and S100A9 can form homodimers (S100A8/ A9) and have manybiological functions [37]. It is noted that S100A8/A9 can activate the vascular endothelium and increase endothelial permeability and has been found associated with atherogenesis and cardiovascular disease [37-39]. S100A8/A9 complex plasma levels were significantly higher in patients with chronic heart failure [40]. It is suggested that serum S100A8/A9 was shown to be associated with the severity of CAD in patients with diabetes mellitus [41, 42]. Moreover, S100A8 showing increased expression in patients with CAD [43]. In this study, it was also found that the up-regulated expression of S100A8 in CAD, which was in line with the previous reports. The present results further demonstrated the crucial role of S100A8 in CAD.

Among the top 10 up-regulated DEGs, matrix metallopeptidase 9 (MMP9) was also found to be related to CAD. It is reported that MMP9 is a gene that associated with atherosclerosis [44]. MMP9 is increased after vascular injury, particularly obvious in inflammatory atherosclerotic lesions [45]. Moreover, the concentration of MMP9 in plasma is a predictor of cardiovascular mortality [46]. Some studies have suggested that the plasma MMP9 is noticeably higher in CAD patients and higher circulating level of MMP9 is related to an adverse prognosis $[47,48]$. In this study, increased expression of MMP9 was found, which is in line with previous reports. This further suggested the role of MMP9 in the process of CAD. However, RT-qPCR result was inconsistent with the integrated analysis and the small sample may be related to this difference. Further research is required.

In this study, three down-regulated DEGs including PRKX, HSPH1 and ZMYND11 in CAD were foundwhich had not been reported in previous studies. Therefore, this result found new CAD-related genes and was facilitating the disclosure of the molecular mechanism underlying CAD. There are limitations to this study. Firstly, the blood samples and number of validated differentially expressed genes in the RT-qPCR were small. Larger numbers of blood samples and larger numbers of genes for validation are needed in further study. Secondly, the data in the GEO database were from leucocytes. And the identified DEGs can be considered as markers of $\mathrm{CAD}$ and were not linked to pathophysiological of CAD. Therefore, the expression of pathological genes in the atherosclerotic lesion also needs testing in further research.

\section{Conclusions}

In summary, it was found that 7 DEGs including IL1R2, ARRB2, PRKX, PLAUR, HSPH1, ZMYND11 and S100A8 may play essential roles in CAD. Additionally, the signaling pathway of MAPK may be a crucial biological signal in the pathology of $\mathrm{CAD}$.

\section{Conflict of interest: None declared}

\section{References}

1. Chen CL, Chen L, Yang WC. The influences of Taiwan's generic grouping price policy on drug prices and expenditures: evidence from analysing the consumption of the three most-used classes of cardiovascular drugs. BMC Public Health. 2008; 8: 118, doi: 10.1186/14712458-8-118, indexed in Pubmed: 18405385.

2. Murray CJ, Lopez AD. Alternative projections of mortality and disability by cause 1990-2020: Global Burden of Disease Study. Lancet. 1997; 349(9064): 1498-1504, doi: 10.1016/S0140-6736(96)07492-2, indexed in Pubmed: 9167458.

3. Opstad TB, Arnesen H, Pettersen A , et al. The MMP-9 -1562 C/T polymorphism in the presence of metabolic syndrome increases the risk of clinical events in patients with coronary artery disease. PLoS One. 2014; 9(9): e106816, doi: 10.1371/journal.pone.0106816, indexed in Pubmed: 25191702.

4. Yusuf S, Hawken S, Ounpuu S, et al. Effect of potentially modifiable risk factors associated with myocardial infarction in 52 countries (the INTERHEART study): case-control study. Lancet. 2004; 364(9438): 937-952, doi: 10.1016/S0140-6736(04)17018-9, indexed in Pubmed: 15364185.

5. Deb S, Wijeysundera HC, Ko DT, et al. Coronary artery bypass graft surgery vs percutaneous interventions in coronary revascularization: a systematic review. JAMA. 2013; 310(19): 2086-2095, doi: 10.1001/ jama.2013.281718, indexed in Pubmed: 24240936.

6. Yang Y, Yang W, Huo W, et al. Identification of biomarkers for ischemic cardiomyopathy based on microarray data analysis. Cardiol J 2017; 24(3): 305-313, doi: 10.5603/CJ.a2017.0005, indexed in Pubmed: 28150292.

7. Marot G, Foulley JL, Mayer CD, et al. Moderated effect size and P-value combinations for microarray meta-analyses. Bioinformatics. 2009; 25(20): 2692-2699, doi: 10.1093/bioinformatics/btp444, indexed in Pubmed: 19628502.

8. Wrighton KH, Lin X, Yu PB, et al. Transforming Growth Factor \{beta\} Can Stimulate Smad1 Phosphorylation Independently of Bone Morphogenic Protein Receptors. J Biol Chem. 2009; 284(15): 9755-9763, doi: 10.1074/jbc.M809223200, indexed in Pubmed: 19224917.

9. Chatr-Aryamontri A, Oughtred R, Boucher L, et al. The BioGRID interaction database: 2015 update. Nucleic Acids Res. 2015; 43(Database issue): D470-D478, doi: 10.1093/nar/gku1204, indexed in Pubmed: 25428363.

10. Shannon P, Markiel A, Ozier O, et al. Cytoscape: a software environment for integrated models of biomolecular interaction networks. Genome Res. 2003; 13(11): 2498-2504, doi: 10.1101/gr.1239303, indexed in Pubmed: 14597658.

11. Stelzl U, Worm U, Lalowski M, et al. A human protein-protein interaction network: a resource for annotating the proteome. Cell. 2005; 122(6): 957-968, doi: 10.1016/j.cell.2005.08.029, indexed in Pubmed: 16169070 . 
12. Zhang Y, Tang W, Peng L, et al. Identification and validation of microRNAs as endogenous controls for quantitative polymerase chain reaction in plasma for stable coronary artery disease. Cardiol J. 2016; 23(6): 694-703, doi: 10.5603/CJ.2016.0109, indexed in Pubmed: 27976798.

13. Libby P. Inflammation in Atherosclerosis. Arterioscler Thromb Vasc Biol. 2012; 32(9): 2045-2051, doi: 10.1161/atvbaha.108.179705.

14. Seger R, Krebs EG. The MAPK signaling cascade. FASEB J. 1995; 9(9): 726-735, indexed in Pubmed: 7601337.

15. Yabluchanskiy A, Ma Y, DeLeon-Pennell KY, et al. Myocardial Infarction Superimposed on Aging: MMP-9 Deletion Promotes M2 Macrophage Polarization. J Gerontol A Biol Sci Med Sci. 2016; 71(4): 475-483, doi: 10.1093/gerona/glv034, indexed in Pubmed: 25878031.

16. Park HJ, Noh JiH, Eun JW, et al. Assessment and diagnostic relevance of novel serum biomarkers for early decision of ST-elevation myocardial infarction. Oncotarget. 2015; 6(15): 12970-12983, doi: 10.18632/ oncotarget.4001, indexed in Pubmed: 26025919.

17. Bondar G, Cadeiras M, Wisniewski N, et al. Comparison of whole blood and peripheral blood mononuclear cell gene expression for evaluation of the perioperative inflammatory response in patients with advanced heart failure. PLoS One. 2014; 9(12): e115097, doi: 10.1371/ journal.pone.0115097, indexed in Pubmed: 25517110.

18. Jickling GC, Xu H, Stamova B, et al. Signatures of cardioembolic and large-vessel ischemic stroke. Ann Neurol. 2010; 68(5): 681-692, doi:10.1002/ana.22187, indexed in Pubmed: 21031583.

19. McGeachie M, Ramoni RL, Mychaleckyj JC, et al. Integrative predictive model of coronary artery calcification in atherosclerosis. Circulation. 2009; 120(24): 2448-2454, doi: 10.1161/CIRCULATIONAHA.109.865501, indexed in Pubmed: 19948975.

20. Zhang H, Li T, Wu G, et al. Integration of partial least squares and Monte Carlo gene expression analysis in coronary artery disease. Exp Ther Med. 2014; 7(5): 1151-1154, doi: 10.3892/etm.2014.1610, indexed in Pubmed: 24940402.

21. Gu S, Su P, Yan J, et al. Comparison of gene expression profiles and related pathways in chronic thromboembolic pulmonary hypertension. Int J Mol Med. 2014; 33(2): 277-300, doi: 10.3892/ijmm.2013.1582, indexed in Pubmed: 24337368

22. Liu Z, Zhou C, Liu Y, et al. The expression levels of plasma micoRNAs in atrial fibrillation patients. PLoS One. 2012; 7(9): e44906, doi: 10.1371/journal.pone.0044906, indexed in Pubmed: 23028671.

23. Liu H, Qin H, Chen Gx, et al. Comparative expression profiles of microRNA in left and right atrial appendages from patients with rheumatic mitral valve disease exhibiting sinus rhythm or atrial fibrillation. J Translat Med. 2014; 12(1): 90, doi: 10.1186/1479-5876-12-90.

24. Devaux Y, Bousquenaud M, Rodius S, et al. Transforming growth factor beta receptor 1 is a new candidate prognostic biomarker after acute myocardial infarction. BMC Med Genomics. 2011; 4: 83, doi: 10.1186/1755-8794-4-83, indexed in Pubmed: 22136666.

25. Ip JE, Wu Y, Huang J, et al. Mesenchymal stem cells use integrin beta1 not CXC chemokine receptor 4 for myocardial migration and engraftment. Mol Biol Cell. 2007; 18(8): 2873-2882, doi: 10.1091/mbc. E07-02-0166, indexed in Pubmed: 17507648.

26. Masud R, Shameer K, Dhar A, et al. Gene expression profiling of peripheral blood mononuclear cells in the setting of peripheral arterial disease. J Clin Bioinforma. 2012; 2: 6, doi: 10.1186/2043-9113-2-6, indexed in Pubmed: 22409835

27. Robless PA, Okonko D, Lintott P, et al. Increased platelet aggregation and activation in peripheral arterial disease. Eur J Vasc Endovasc Surg. 2003; 25(1): 16-22, indexed in Pubmed: 12525806.

28. Huang CC, Lloyd-Jones DM, Guo X, et al. Gene expression variation between African Americans and whites is associated with coronary artery calcification: the multiethnic study of atherosclerosis. Physiol Genomics. 2011; 43(13): 836-843, doi: 10.1152/physiolgenomics.00243.2010, indexed in Pubmed: 21521779.

29. Wilensky RL, Shi Yi, Mohler ER, et al. Inhibition of lipoprotein-associated phospholipase A2 reduces complex coronary atherosclerotic plaque development. Nat Med. 2008; 14(10): 1059-1066, doi: 10.1038/ nm.1870, indexed in Pubmed: 18806801.

30. Elashoff MR, Wingrove JA, Beineke $\mathrm{P}$, et al. Development of a blood-based gene expression algorithm for assessment of obstructive coronary artery disease in non-diabetic patients. BMC Med
Genomics. 2011; 4: 26, doi: 10.1186/1755-8794-4-26, indexed in Pubmed: 21443790.

31. Grover MP, Ballouz S, Mohanasundaram KA, et al. Novel therapeutics for coronary artery disease from genome-wide association study data. BMC Med Genomics. 2015; 8 Suppl 2: S1, doi: 10.1186/1755-8794-8S2-S1, indexed in Pubmed: 26044129.

32. Dimitrakopoulou K, Vrahatis AG, Bezerianos A. Integromics network meta-analysis on cardiac aging offers robust multi-layer modular signatures and reveals micronome synergism. BMC Genomics. 2015; 16 147, doi: 10.1186/s12864-015-1256-3, indexed in Pubmed: 25887273.

33. Sharma R, Zhou MM. Partners in crime: The role of tandem modules in gene transcription. Protein Sci. 2015; 24(9): 1347-1359, doi: 10.1002/pro.2711, indexed in Pubmed: 26059070.

34. Kurozumi K, Nishita M, Yamaguchi K, et al. BRAM1, a BMP receptorassociated molecule involved in BMP signalling. Genes Cells. 1998; 3(4): 257-264, indexed in Pubmed: 9663660.

35. Wen H, Li Y, Xi Y, et al. ZMYND11 links histone H3.3K36me3 to transcription elongation and tumour suppression. Nature. 2014; 508(7495): 263-268, doi: 10.1038/nature13045, indexed in Pubmed: 24590075.

36. Ansieau S, Sergeant A. [BS69 and RACK7, a potential novel class of tumor suppressor genes]. Pathol Biol (Paris). 2003; 51(7): 397-399, indexed in Pubmed: 12948759.

37. Cotoi OS, Dunér P, Ko N, et al. Plasma S100A8/A9 correlates with blood neutrophil counts, traditional risk factors, and cardiovascular disease in middle-aged healthy individuals. Arterioscler Thromb Vasc Biol. 2014; 34(1): 202-210, doi: 10.1161/ATVBAHA.113.302432, indexed in Pubmed: 24202303.

38. Viemann D, Strey A, Janning A, et al. Myeloid-related proteins 8 and 14 induce a specific inflammatory response in human microvascular endothelial cells. Blood. 2005; 105(7): 2955-2962, doi: 10.1182 blood-2004-07-2520, indexed in Pubmed: 15598812.

39. Viemann D, Barczyk K, Vogl T, et al. MRP8/MRP14 impairs endothelial integrity and induces a caspase-dependent and -independent cell death program. Blood. 2007; 109(6): 2453-2460, doi: 10.1182/ blood-2006-08-040444, indexed in Pubmed: 17095618.

40. Imbalzano E, Mandraffino G, Casciaro M, et al. Pathophysiological mechanism and therapeutic role of S100 proteins in cardiac failure: a systematic review. Heart Fail Rev. 2016; 21(5): 463-473, doi: 10.1007/ s10741-016-9529-8, indexed in Pubmed: 26833319.

41. Nagareddy PR, Murphy AJ, Stirzaker RA, et al. Hyperglycemia promotes myelopoiesis and impairs the resolution of atherosclerosis. Cell Metab. 2013; 17(5): 695-708, doi: 10.1016/j.cmet.2013.04.001, indexed in Pubmed: 23663738.

42. Peng WH, Jian WX, Li HL, et al. Increased serum myeloid-related protein $8 / 14$ level is associated with atherosclerosis in type 2 diabetic patients. Cardiovasc Diabetol. 2011; 10: 41, doi: 10.1186/1475-284010-41, indexed in Pubmed: 21592353.

43. Poduri A, Bahl A, Talwar KK, et al. Proteomic analysis of circulating human monocytes in coronary artery disease. Mol Cell Biochem. 2012; 360(1-2): 181-188, doi: 10.1007/s11010-011-1055-3, indexed in Pubmed: 21938407.

44. Blin J, Ahmad Z, Rampal LR, et al. Preliminary assessment of differential expression of candidate genes associated with atherosclerosis. Genes Genet Syst. 2013; 88(3): 199-209, indexed in Pubmed: 24025248.

45. Bendeck MP, Zempo N, Clowes AW, et al. Smooth muscle cell migration and matrix metalloproteinase expression after arterial injury in the rat. Circ Res. 1994; 75(3): 539-545, indexed in Pubmed: 8062427.

46. Ye S. Influence of matrix metalloproteinase genotype on cardiovascular disease susceptibility and outcome. Cardiovasc Res. 2006 69(3): 636-645, doi: 10.1016/j.cardiores.2005.07.015, indexed in Pubmed: 16122719 .

47. Kai $\mathrm{H}$, Ikeda $\mathrm{H}$, Yasukawa $\mathrm{H}$, et al. Peripheral blood levels of matrix metalloproteases- 2 and -9 are elevated in patients with acute coronary syndromes. J Am Coll Cardiol. 1998; 32(2): 368-372, indexed in Pubmed: 9708462.

48. Blankenberg S, Rupprecht HJ, Poirier O, et al. AtheroGene Investigators. Plasma concentrations and genetic variation of matrix metalloproteinase 9 and prognosis of patients with cardiovascular disease. Circulation. 2003; 107(12): 1579-1585, doi: 10.1161/01. CIR.0000058700.41738.12, indexed in Pubmed: 12668489. 